\title{
Kombination von wasserbaulichem Modellversuch mit numerischer Strömungsberechnung gezeigt am Beispiel des BHQ-Abflusses im Stauraum des Kraftwerks Ashta 1
}

\begin{abstract}
Zusammenfassung: Inhalt dieses Beitrages ist die Beschreibung von hydraulischen Modellversuchen und die Gegenüberstellung mit den Ergebnissen der numerischen Simulation des Bemessungshochwasserabflusses (BHQ $=5900 \mathrm{~m}^{3} / \mathrm{s}$, entspricht einem geschätzten 500-jährlichen Ereignis) im Stauraum der geplanten HydromatrixKraftwerksanlage Ashta 1 in Albanien. Im Rahmen der Projektierung hatte der Planer Pöyry Energy unter anderem auch eine 2dBerechnung dieses Extremereignisses durchgeführt. Aufgrund der massiven Auswirkung der Hochwasserstände auf die weitere Planung, und der Bedeutung für eine angrenzende Siedlung und eine Hauptverkehrsverbindung, wurde auch das Institut für Wasserbau und Ingenieurhydrologie der TU Wien mit der Durchführung von hydraulischen Modellversuchen als Grundlage und zur Absicherung dieser numerischen Berechnungen beauftragt. Die Resultate der unabhängig voneinander durchgeführten Untersuchungen werden hier vorgestellt.
\end{abstract}

Combining hydraulic model tests with numerical flow analysis: The design flood for the reservoir above the Ashta 1 power station in Albania

Summary: This article describes hydraulic scale model tests and compares the results with those obtained from a numerical simulation of the design flood $\left(5,900 \mathrm{~m}^{3} / \mathrm{s}\right.$ corresponding to an estimated 500-year event) for the reservoir of the planned Ashta 1 Hydromatrix power development in Albania. The investigation led by Pöyry Energy, included a 2d analysis of this extreme event. Considering the substantial effect the calculated results will have on further planning and design as well as the importance of the flood water levels for a neighbouring residential area and a main traffic connection, a contract was awarded to the Institute of Hydraulic and Water

Institut für Wasserbau und Ingenieurhydrologie, TU Wien

2 Pöyry Energy GmbH, Wien

Verbund - AHP AG, Wien

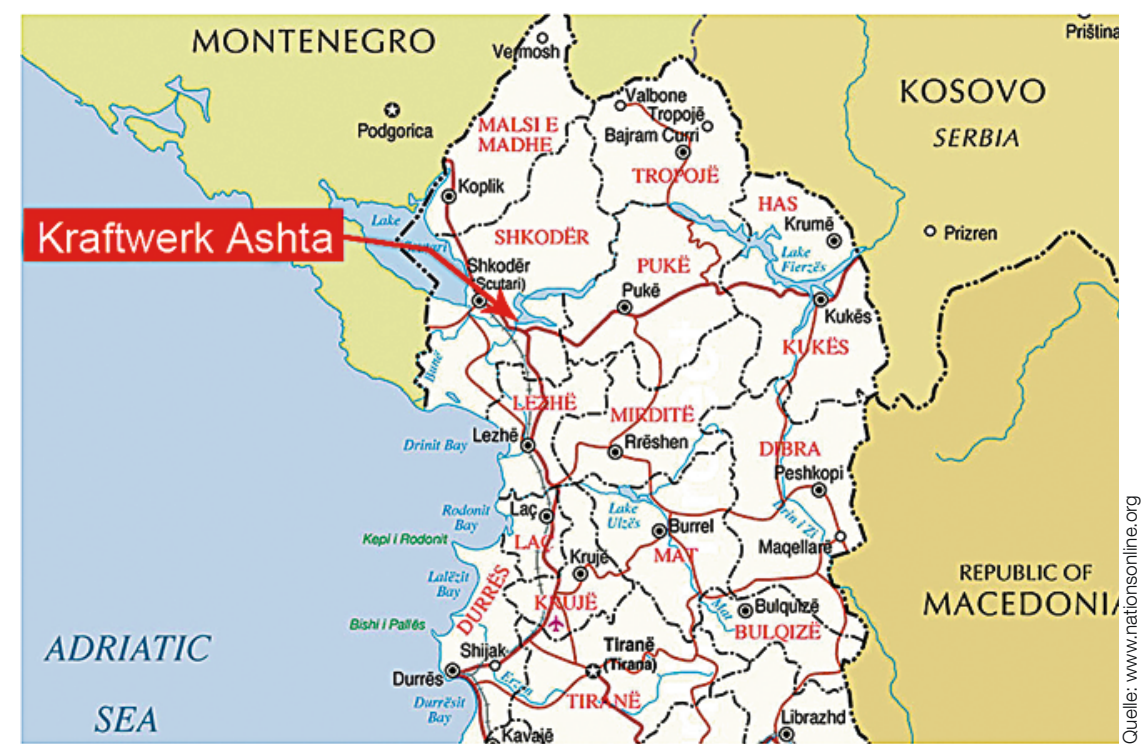

Abb. 1: Geographische Lage der KW-Anlage Ashta.

Resources Engineering at the Vienna University of Technology for performing hydraulic scale model tests as a basis for these numerical analyses and for checking the results. This article presents the results of the two independently-conducted studies.

\section{Einleitung}

Der Verbund bzw. seine Tochterfirma Verbund International plant in Albanien das weltgrößte Hydromatrix-Kraftwerk als BOT - Projekt (build - operate - transfer). Bis 2012 soll die Projektgesellschaft „Energji Ashta" eine 48 MW starke Wasserkraftanlage im Norden Albaniens (Abb. 1), nahe der Stadt Shkodra am Drin - Fluss fertigstellen. Mit der künftig jährlich zu erwarteten Stromerzeugung von ca. $230 \mathrm{GWh}$ können 100000 albanische Haushalte mit Elektrizität versorgt werden.

\subsection{Projektbeschreibung}

Aus bautechnischen Gründen und aus Belangen des Umweltschutzes ist die Anlage Ashta linksufrig des Drin - Flusses als zweistufiges Ausleitungskraftwerk konzi- piert, wobei die Oberstufe Ashta 1 das bereits 1972 erbaute Unterwasserbecken der Stauanlage Vau I Dejes mit dem $3 \mathrm{~km}$ unterhalb liegenden Spathara Wehr als Stauraum nutzt. Die Anlage liegt am Ende einer bestehenden fünfstufigen Kraftwerkskette, das Stauziel wird bei Ashta 1 auf Kote $23.00 \mathrm{~m}$ ü. A. festgelegt und soll durch den Einbau eines Schlauchwehrs erreicht werden. Die Ausbauwassermenge von $560 \mathrm{~m}^{3} / \mathrm{s}$ wird über das Krafthaus von Ashta 1 über 45 Hydromatrix - Einheiten bei einer durchschnittlichen Fallhöhe von ca. 5,30 m abgearbeitet. Unterwasserseitig erfolgt der Anschluss über einen ca. $5 \mathrm{~km}$ langen Triebwasserkanal an das Krafthaus der zweiten Stufe Ashta 2, wo ebenfalls 45 Hydromatrix - Einheiten installiert sind, und den Rückgabekanal in den Drin Fluss ( $A b b$. 2).

Das Hochwasser wird über das bestehende Spathara Wehr mit 15 jeweils ca. $14,5 \mathrm{~m}$ breiten Wehrfeldern (Abb. 3) abgeführt.

\subsection{Problemstellung}

Ziel der Untersuchungen war es, die Hochwasserabfuhrfähigkeit der bestehenden Wehranlage Spathara unter Berücksichtigung des geplanten Schlauchwehrs für ei- 


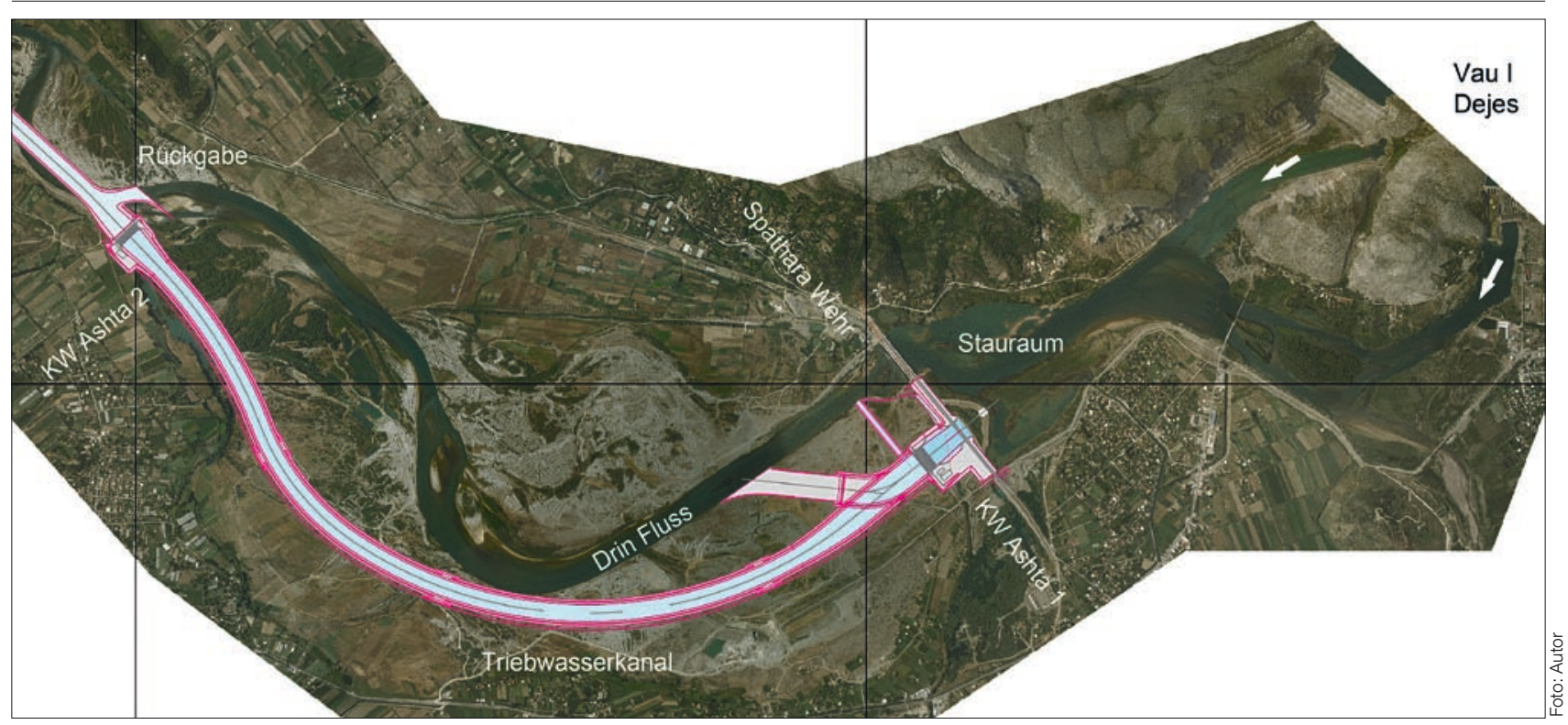

Abb. 2: Genereller Übersichtslageplan der gesamten Kraftwerksanlage Ashta.

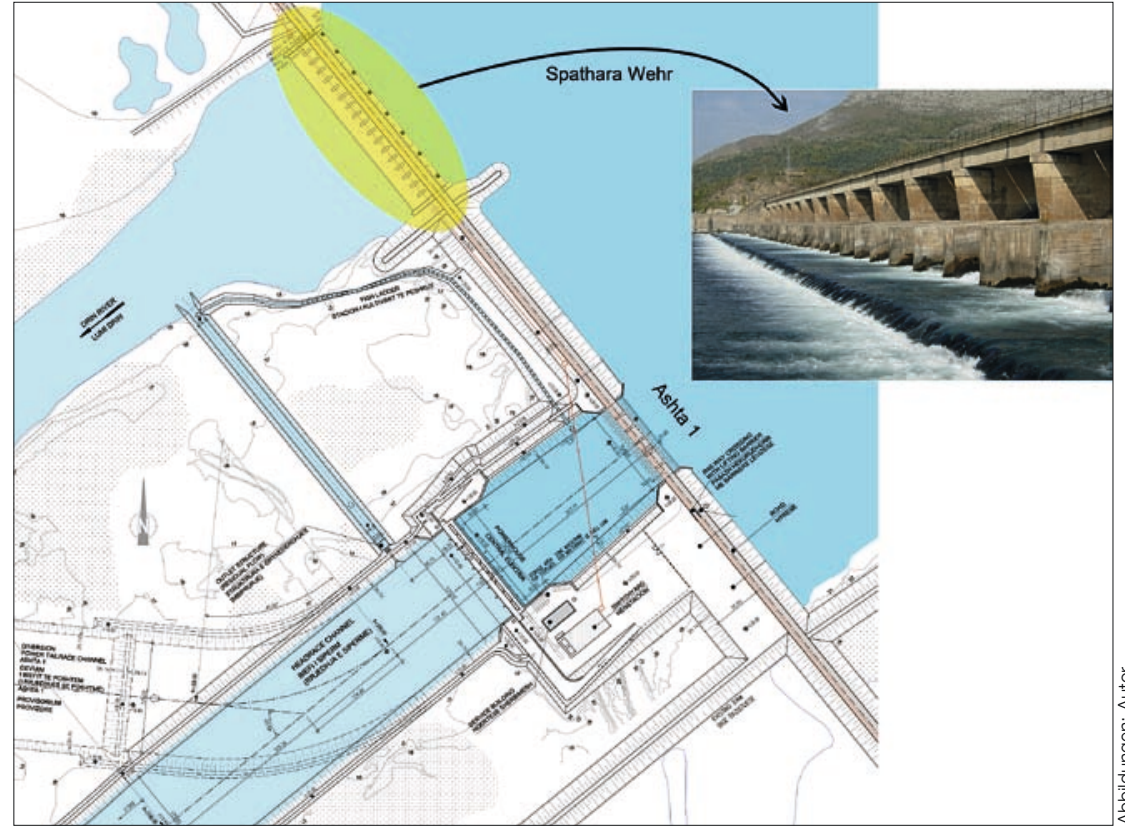

nen Bemessungsabfluss von $5900 \mathrm{~m}^{3} / \mathrm{s}$ nachzuweisen, ohne dass es zu einem Einstau oder einer Überflutung des über diese Wehranlage führenden Eisenbahn- und Straßenbrückentragwerkes kommt.

Weiters war zu prüfen, ob der Wasserspiegel während des Bemessungsereignisses am linken Begleitdamm im Bereich der dahinterliegenden Siedlung die Marke 27,0 mü. A. übersteigt. Vorgesehen ist eine Dammhöhe von mindestens 28,0 mü.A. Bei Nichteinhaltung dieser Forderungen wären zusätzliche bauliche Maßnahmen wie Baggerungen im Stauraum, Dammerhöhungen und eventuell auch der Bau einer zusätzlichen Hochwasserentlastungsanlage erforderlich.

Im Zuge der Projektierung wurde der maßgebende Hochwasserabfluss im betroffenen Gebiet mit einer 2d-Berechnung von Pöyry simuliert. Aufgrund des hohen Gefährdungspotentials wurde das Institut für Wasserbau und Ingenieurhydrologie

Abb. 3: Lageplan KW Ashta 1 mit UW Ansicht der bestehenden Wehranlage Spathara. der TU Wien mit der Durchführung von

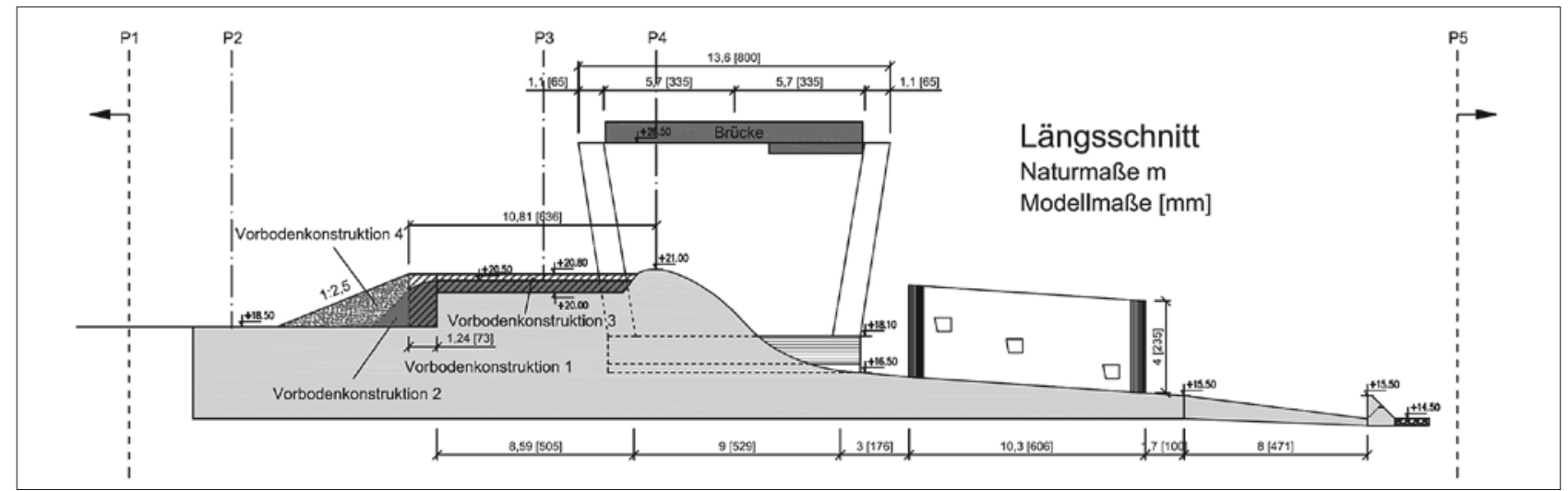

Abb. 4: Längsschnitt des Schnittmodells mit verschiedenen Vorbodenkonstruktionen für das Schlauchwehr auf dem bestehenden Wehr. 


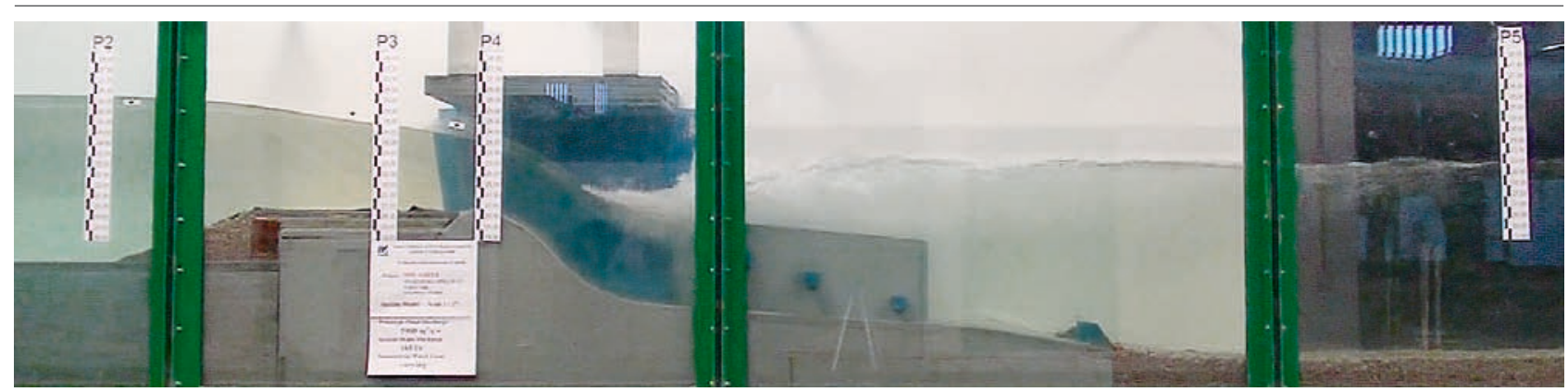

Abb. 5: Modellansicht bei $\mathrm{BHQ}=5900$ m³/s (im Modell $165 \mathrm{l} / \mathrm{s}$ ).

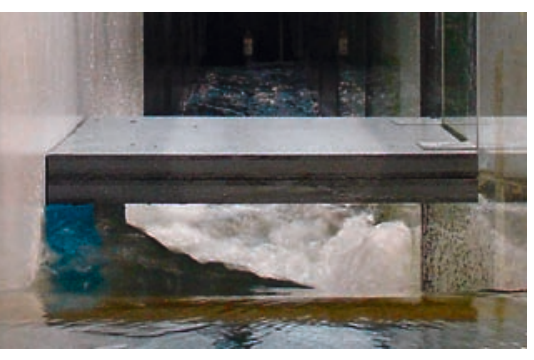

Abb. 6: Oberwasseransicht der Pfeilerstauwelle bei $\mathrm{BHQ}$

zwei hydraulischen Modellversuchen zur Absicherung der Berechnungsergebnisse beauftragt.

In einem ersten Schritt wurde mit Hilfe eines hydraulischen Schnittmodells im Maßstab 1:17 die Hochwasserabfuhrfähigkeit des bestehenden Spathara Wehrs und der mittels Schlauchwehr ausgebauten Wehranlage bestimmt. Die Ergebnisse dieser Untersuchung definierten die unterwasserseitige Randbedingung der numerischen Berechnung. In einem weiteren Schritt sollten mit Hilfe eines hydraulischen Vollmodells im Maßstab 1:50 die numerischen Ergebnisse abgesichert, und auch eine bessere Beurteilung der hydrodynamischen Prozesse im Stauraum ermöglicht werden.

\section{Hydraulisches Schnittmodell}

Das Schnittmodell wurde in einer Glasrinne des Wasserbaulabors im Modellmaßstab von 1:17 errichtet und bildete ein halbes Wehrfeld und einen halben Wehrpfeiler nach. Für den Bemessungsabfluss von $5900 \mathrm{~m}^{3} / \mathrm{s}$, bei einer freien Durchflussbreite von $218 \mathrm{~m}$ ohne Wehrpfeiler ergibt sich ein spezifischer Abfluss von $27 \mathrm{~m}^{2} / \mathrm{s}$. Die Umrechnung der Naturdaten auf das Schnittmodell und vice versa erfolgte mit Hilfe des Froude'schen Modellgesetzes.

Im Zuge der Versuche am Schnittmodell wurden mehrere Vorbodenkonstruktionen zur Auflage des Schlauchwehrs (Abb. 4) und ihre Auswirkung auf die Wasserspiegellagen oberhalb der Wehranlage getestet, dabei wurden die Wasserstände in fünf Querschnitten (P1 bis P5) gemessen (Tab. 1). Nachfolgend werden nur die Ergebnisse der Untersuchungen des Ausführungsvorschlages präsentiert.

\subsection{Ausbildung der Schlauchwehrplatte}

Die gewählte Ausführungsvariante weist eine hydraulisch günstige Gestaltung des Vorbodens auf Kote 20,80 m ü.A. mit einer ca. 1:2,5 geneigten betonierten Anrampung (breites Wehr) auf (Abb. 4). Damit sollte der durch das Wehr initiierte oberwasserseitige Rückstau reduziert werden, sowie der Freibord unterhalb der Brücke vergrößert und auch bautechnische Erfordernisse erfüllt werden.

\subsection{Versuchsergebnisse Schnittmodell}

Die Versuche zeigten, dass für den Bemessungsabfluss (BHQ) von $5900 \mathrm{~m}^{3} / \mathrm{s}$ bis zu einem Unterwasserpegel auf Kote 23,5 m ü. A. jedenfalls ein vollkommener Überfall über das Wehr vorliegt. Dieser Grenzwert wird nach dem berechneten Unterwasserpegelschlüssel jedoch nicht erreicht, daher stellt sich im Bemessungsfall jedenfalls ein vollkommener Überfall ein (Abb. 5).

Auch im Falle eines Unterwasserspiegelanstieges bis Kote 26,0 mü. A. konnte das Bemessungshochwasser gefahrlos unterhalb der vorhandenen Brückenkonstruktion (Unterkante Kote 26,50 mü.A.) abgeführt werden. Beim Halbpfeiler trat jedoch aufgrund seiner Kontraktionswirkung eine starke Stoßwelle auf, welche nahezu die Brückenunterkante erreichte (Abb. 6).

Die im Schnittmodell gemessenen Wasserspiegel im Oberwasser (P1) liegen auf der unsicheren Seite, da räumliche Einflüsse der Anströmung (Querströmungen, breiter Zulaufbereich, etc.) nicht naturähnlich erfasst werden.

Als Näherung für die Staukote im Oberwasser kann die Energiehöhe aus dem Kontrollquerschnitt P1 berechnet werden. Die Energielinienhöhe in P1 beträgt beim Bemessungsabfluss bei der Ausführungsvariante ca. 26,90 mü. A.

Eine gesicherte Bestimmung der Wasserspiegellage für den Bemessungsfall kann erst anhand eines Vollmodells, in dem räumliche Einflüsse wie asymmetrische Anströmung (Wirbelbildung bei Wehrpfeilerköpfen), Pfeilereinbauten des Schlauchwehrs, etc. entsprechend berücksichtigt sind, erfolgen. Unter den vorliegenden Einschränkungen erlauben die Untersuchungen am Schnittmodell jedoch die grundsätzliche Aussage, dass der Bemessungsabfluss BHQ von $5900 \mathrm{~m}^{3} / \mathrm{s}$ mit hoher Wahrscheinlichkeit gefahrlos über die vorhandenen 15 Wehrfelder abgeführt werden kann, ohne die zulässigen Freibordgrenzen zu überschreiten. Damit erwies sich der Bau einer zusätzlichen Hochwasserentlastung bereits zu diesem Zeitpunkt als entbehrlich.

\section{Numerische \\ Strömungsberechnung}

Wie bereits erwähnt wurden die Hochwasserverhältnisse beim Bemessungsabfluss mittels einer 2d-Berechnung simuliert um Grundlagen für die weitere Planung der

\begin{tabular}{|c|c|c|c|c|c|c|}
\hline$Q_{\text {Natur }}\left[\mathrm{m}^{3} / \mathrm{s}\right]$ & $\mathbf{Q}_{\text {Modell }}[\mathrm{I} / \mathrm{s}]$ & P1 [m] & P2 [m] & P3 [m] & P4 [m] & P5 [m] \\
\hline 5900 & 165 & 26,69 & 26,44 & 25,09 & 24,26 & 22,00 \\
\hline
\end{tabular}




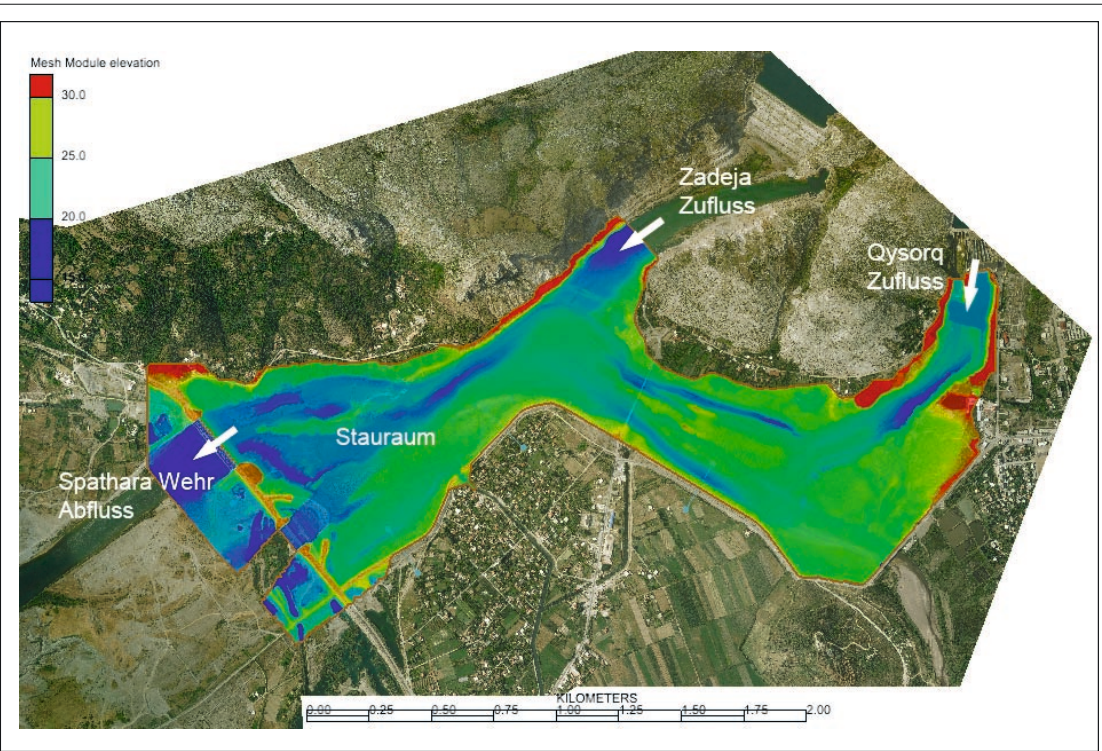

Abb. 7: Übersicht über den 2-d Modellbereich mit Zu- und Abflüssen.

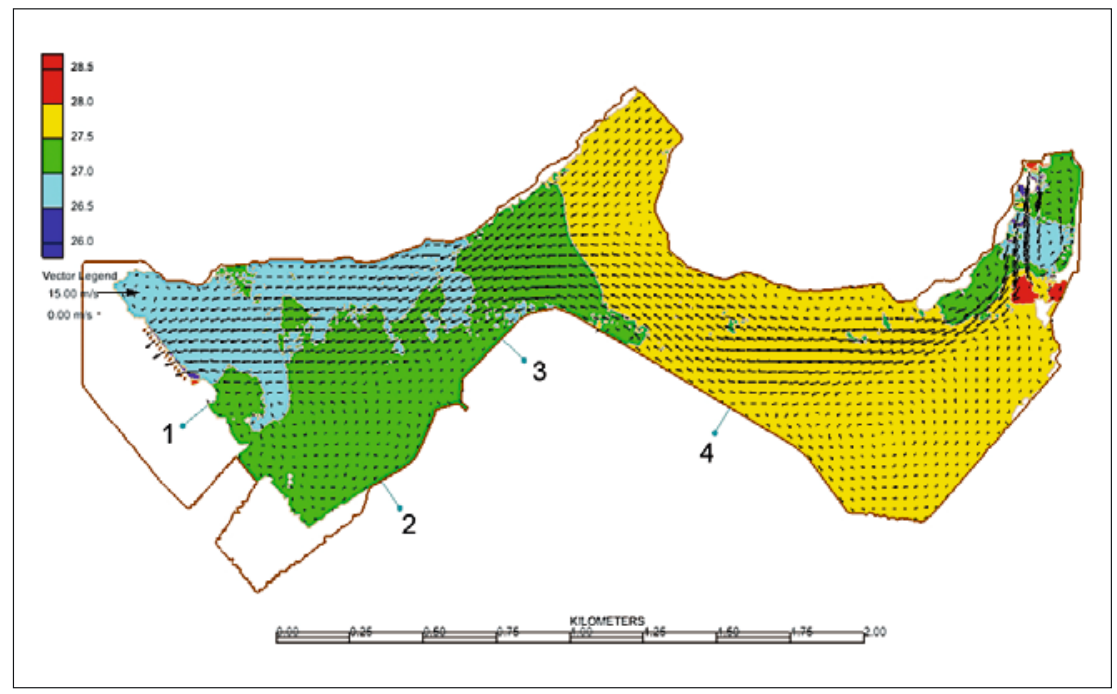

Abb. 8: Berechnete Wasserspiegel und Geschwindigkeitsvektoren beim $B H Q=5900 \mathrm{m3} / \mathrm{s}$.

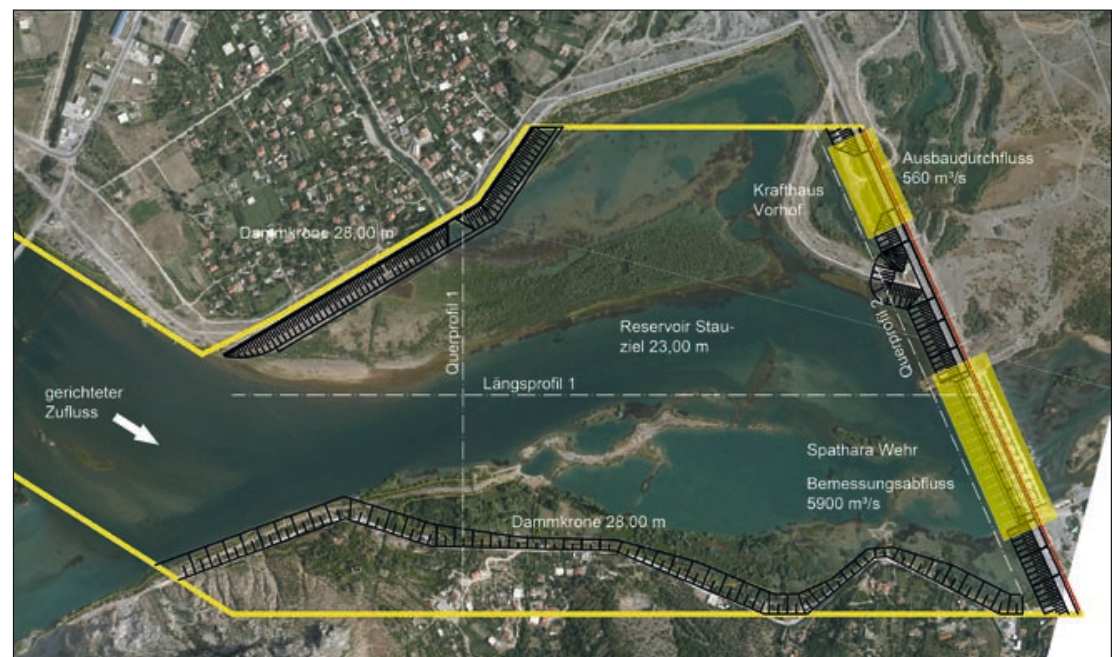

Abb. 9: Bereich des physikalischen Modells.
Anlage zu erhalten. Im Besonderen betraf dies die Hochwassersicherheit des linken Begleitdammes, der eine dahinter liegende Siedlung schützt, und auch die Überflutungssicherheit der Eisenbahnund Straßenbrücke über die bestehende Wehranlage Spathara. Zur Einhaltung dieser Forderungen wurden etwaige Baggerungen im Stauraum in Erwägung gezogen, aber auch der Bau einer zusätzlichen HW-Entlastung. Die Notwendigkeit dieser baulichen Maßnahmen würde jedenfalls die Wirtschaftlichkeit des Projekts beeinträchtigen.

\subsection{Allgemeines}

Die numerischen Berechungen wurden von Pöyry Energy mit dem Programm Hydro_as-2d V2.2 ohne Sedimentmodul also mit fester Sohle durchgeführt. Dieses Programm beruht auf der Lösung der tiefengemittelten Flachwassergleichungen mit hydrostatischer Druckverteilung unter Vernachlässigung der vertikalen $\mathrm{Ge}$ schwindigkeitskomponente mit Hilfe eines Finite Volumen Ansatzes.

\subsection{Berechnungsnetz und Randbedingungen}

Der Modellbereich des Stauraums ist begrenzt durch den Oberlieger Vau I Dejes und am unteren Ende durch das SpatharaWehr und den Einlauf zum Krafthaus Ashta 1 ( $A b b$. 7). Das Berechnungsnetz beinhaltet 46.000 Elemente mit 35.500 Knoten.

Für das Bemessungshochwasser erfolgte der Zufluss von der Zadeja Hochwasserentlastung mit $2145 \mathrm{~m}^{3} / \mathrm{s}$ und von der Qysorq Anlage mit $3755 \mathrm{~m}^{3} / \mathrm{s}$. Der gesamte Zufluss von $5900 \mathrm{~m}^{3} / \mathrm{s}$ wird nur über das Spathara Wehr abgeführt. Als unterwasserseitige Randbedingung wurde der im Querschnitt P3 des Schnittmodells (siehe Pkt. 2) bestimmte Oberwasserspiegel berücksichtigt, da die Simulation ansonsten die Strömungsverhältnisse direkt oberhalb der Wehranlage zu stark vereinfachen würde. Die Rauigkeit im Stauraum wurde mit einem mittleren Stricklerbeiwert von $33 \mathrm{~m}^{1 / 3} / \mathrm{s}$ abgeschätzt, jene für das Schlauchwehr wurde mit $55 \mathrm{~m}^{1 / 3} / \mathrm{s}$ angenommen.

Es sei angemerkt, dass aufgrund fehlender Wasserspiegelaufzeichnungen bei Hochwasserereignissen zur Modellkalibrierung sowie der limitierten Daten der bathymetrischen Stauraumaufnahme die Berechnungsgrundlagen gewisse Defizite aufweisen. Weiters beinhaltet die 2d-Berechnung Vereinfachungen, die natürlich 


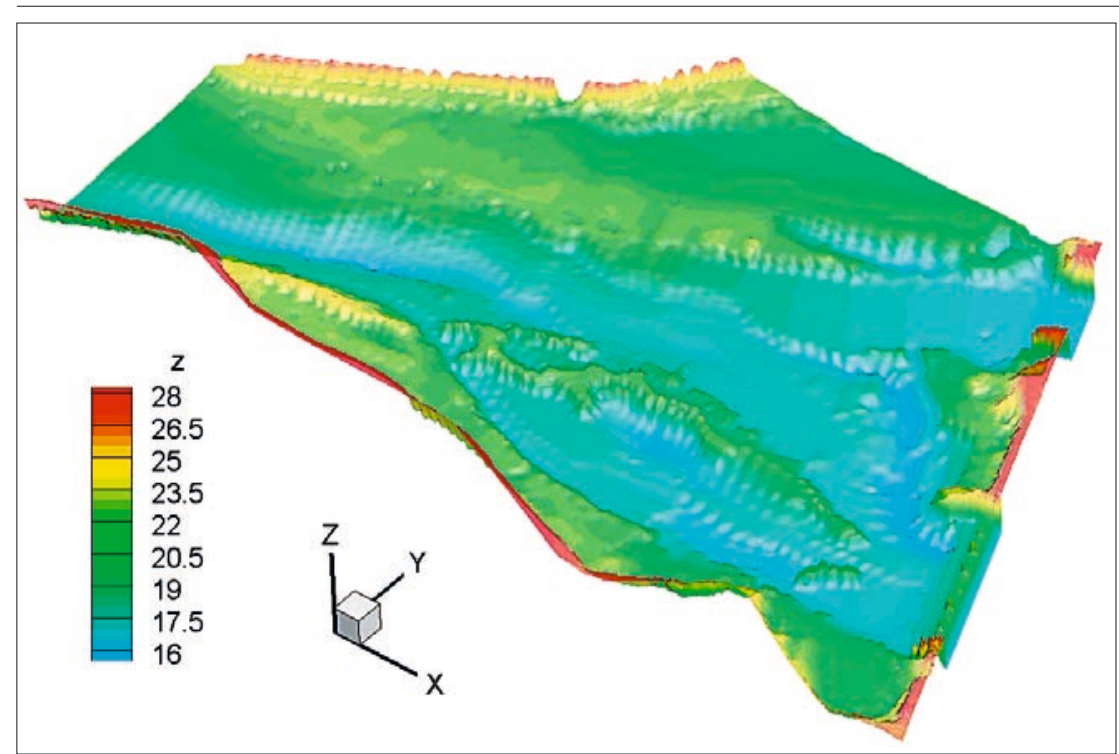

Abb. 10: Generiertes digitales Geländemodell für den Modellaufbau des Stauraums.

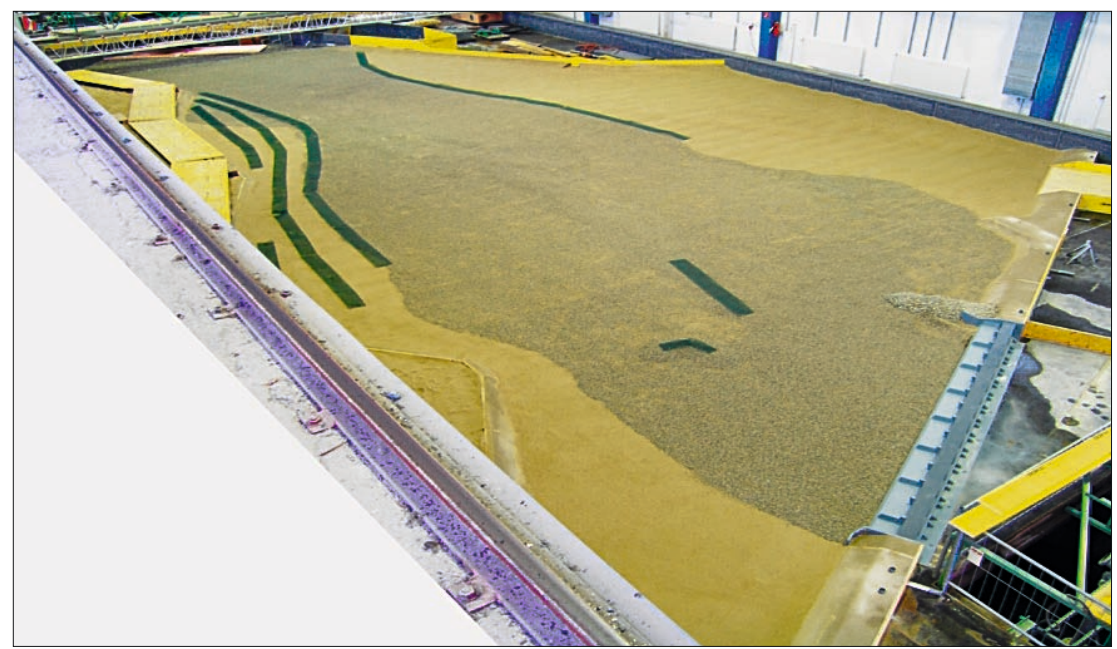

Abb. 11: Vollmodellaufbau mit rauer Sohle.

auch das Berechnungsergebnis mehr oder weniger stark beeinflussen.

\subsection{Ergebnisse}

Die für den Bereich Spathara-Wehr bis Profil $3(A b b .8)$ berechneten Wasserspiegel liegen im Wesentlichen bei 27,0 mü.A., am linksufrigen Damm liegen die Berechnungsergebnisse um wenige $\mathrm{cm}$ darüber. Der verjüngte Fließquerschnitt und die Krümmung stromauf Profil 3 schnüren die Strömung ein, sodass sich vom Profil 4 bis hinauf zum Oberliegerkraftwerk Wasserspiegel von 27,5 bis 28,0 mü. A. einstellen. Der Bereich oberhalb der Krümmung wurde im physikalischen Modell aus Gründen der Modelltechnik und der Kosten nicht nachgebildet, da hier auch nur ein geringes Überflutungsrisiko erwartet wird.
Aufgrund der oben angeführten Unsicherheiten in den Eingangsdaten und Modellparametern sowie den Vereinfachungen in den Berechnungsannahmen sind die Ergebnisse ebenfalls unscharf. Um die Berechnungsergebnisse besser abzusichern, und auch die Dreidimensionalität des Abflussgeschehens besonders durch die vorhandenen angelandeten Inseln naturähnlicher simulieren zu können, wurde im nächsten Schritt ein Vollmodellversuch durchgeführt.

\section{Hydraulisches Vollmodell}

Das Vollmodell des Stauraums der Wasserkraftanlage Ashta 1 wurde zur detaillierten Untersuchung der Wasserspiegellagen im Hochwasserfall (Bemessungshochwasser $5900 \mathrm{~m}^{3} / \mathrm{s}$ ) im Maßstab 1:50 errichtet. Die Abmessungen des gesamten Modellauf- baus betrugen ca. 33 x 16 m, damit konnte der hydraulisch relevante Untersuchungsbereich in der Natur oberwasserseitig der Wehranlage mit einer Länge von ca. $1250 \mathrm{~m}$ und einer Breite von ca. 800 m nachgebildet werden ( $A b b$. 9). Der linksufrige Stauraumrand (Stillwasserbereich) konnte aus Platzgründen in der Versuchshalle nicht berücksichtigt werden. Diese Vereinfachung ist jedoch für die Ergebnisse der vorliegenden Untersuchung ohne Einfluss.

\subsection{Modellaufbau}

Das Modell wurde mit beweglicher Sohle aus Feinsand hergestellt um auch Sedimentumlagerungen qualitativ beurteilen zu können. Weiters sollte auch die Möglichkeit einer raschen Modelladaptierung im Falle von erforderlichen Stauraumbaggerungen zur Verbesserung der Anströmungsverhältnisse zur bestehenden Wehranlage offen gehalten werden. Das digitale dreidimensionale Geländemodell des Stauraums beruht auf einer aktuellen, im Sommer 2009 erstellten bathymetrischen Aufnahme, wobei die geplanten Veränderungen (Baggerungen) im Krafthauszulaufbereich bereits entsprechend berücksichtigt wurden (Abb. 10). Die Formgebung des Stauraumgeländes erfolgte naturgetreu mit Hilfe einer computergesteuerten (Numeric ControlTechnik) Sandfräse.

Die Stauraumsohle war seitlich mit betonierten Dammböschungen (Oberkante 28,00 mü. A.) begrenzt. Unterwasserseitig erfolgte der Abschluss des Vollmodells durch die Wehranlage, die seitlichen Anschlussdämme und den Einlauf zum Hydromatrix-Krafthausvorhof. Im Detail wurde die bestehende Spathara Wehranlage mit Überfall und Pfeilern, das geplante Schlauchwehr (Schlauchwehrpfeiler mit Vorbodenkonstruktion) und der oberwasserseitige Stauraum, jedoch ohne Tosbecken, modelliert (Abb. 11). Der Krafthauszulauf verfügte über eine Auslassöffnung zur kontrollierten Turbinenwasserabgabe von $560 \mathrm{~m}^{3} / \mathrm{s}$ (32 l/s im Modell), und diente auch als zusätzliche Hochwasserabfuhrmöglichkeit für ca. $720 \mathrm{~m}^{3} / \mathrm{s}$ (41 1/s im Modell).

\subsection{Rauigkeitsverhältnisse}

Aufgrund fehlender Daten von Sieblinien im Stauraum, gemessenen Wasserspiegellagen und Abflüssen im Stauraumbereich zur Modellkalibrierung wurde die Sohlrauigkeit so angenähert, dass in einem ersten Versuchsaufbau durch den Einbau 
eines sehr feinen Modellsandes (Feinsand 0,2-2 mm) mit geringer Oberflächenrauigkeit (glatte Sohlausführung ohne Bewuchs) eher tiefe Wasserspiegel erwartet wurden. Ein zweiter Versuchsaufbau erfolgte mit einer Kiesbelegung (gebrochener Kies 2-8 mm) im Bereich der Hauptströmung mit einer sehr hohen Oberflächenrauigkeit und der Nachbildung von Bewuchs am Ufersaum und auf Inselbereichen (mit Drahtgitternetz), von dem sehr hohe Wasserspiegellagen erwartet wurden. Mit diesem Spektrum von Oberflächenrauigkeiten sollte der Einfluss auf die Wasserspiegellage im Hochwasserfall gezeigt werden. Der Einfluss der Wandrauigkeiten der betonierten Uferlinien wurde durch entsprechende raue Ausbildung der Betonoberfläche berücksichtigt, hatte aber keinen Einfluss auf den Abfluss, da die Ufer im Wesentlichen nicht von der Hauptströmung berührt waren.

\subsection{Modellbetrieb und Messtechnik}

Das Modell wurde ebenfalls nach dem Froude'schen Ähnlichkeitsgesetz betrieben, dieses erforderte für das Bemessungshochwasser von $5900 \mathrm{~m}^{3} / \mathrm{s}$ einen Durchfluss von ca. $\mathrm{Q}=334 \mathrm{l} / \mathrm{s}$ im Modell. Die Wasserversorgung mit induktiver Durchflussmessung erfolgte entsprechend dem Hochwasserfall mit dem größten Teil des Abflusses aus der Richtung der Hochwasserentlastung Qysorq des Oberliegerkraftwerkes Vau I Dejes. Nach einem Einlaufbereich mit Strömungsberuhigung war eine mit gröberen Steinen befestigte Übergangszone angeordnet, ehe der eigentliche Modellbereich durchströmt wurde. Der Hochwasserabfluss erfolgte nur über die Wehranlage, der Krafthausauslass blieb geschlossen.

Mittels eines computergesteuerten Messwagens konnte der gesamte Modellbereich in drei Achsen frei angesteuert werden. Diese Messplattform war mit der Sandfräse zur Geländegestaltung, sowie mit triangulierenden Lasersonden zur Gerinnesohlevermessung, mit einer Ultraschallsonde zur Wasserspiegelmessung sowie einer ADV-Sonde zur Geschwindigkeitsmessung ausgerüstet. Bedient wurden alle Messsysteme von einem zentralen Steuerplatz seitlich am Messwagen. Zur unmittelbaren Strömungsvisualisierung wurden leuchtende Schwimmkörper verwendet, welche durch die entsprechende Aufnahmetechnik die Stromlinien mit deren Geschwindigkeiten im Hochwasserfall anschaulich wiedergeben.

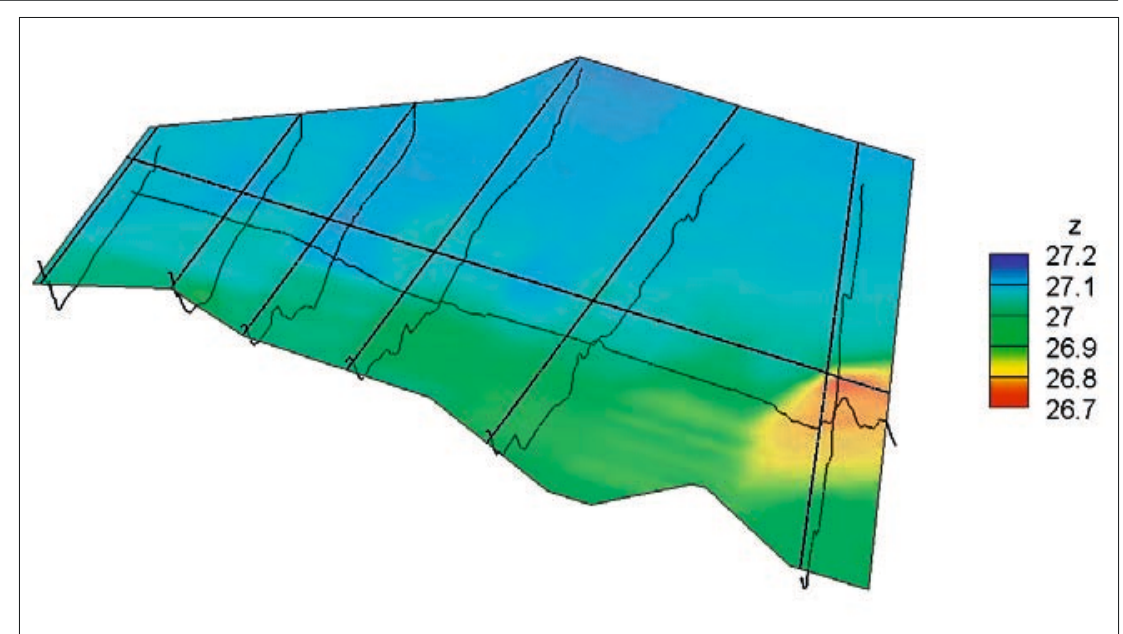

Abb. 12: Wasserspiegellage ( $\mathrm{z}$ in $\mathrm{m})$ bei $\mathrm{BHQ}$ im untersuchten Stauraumbereich.

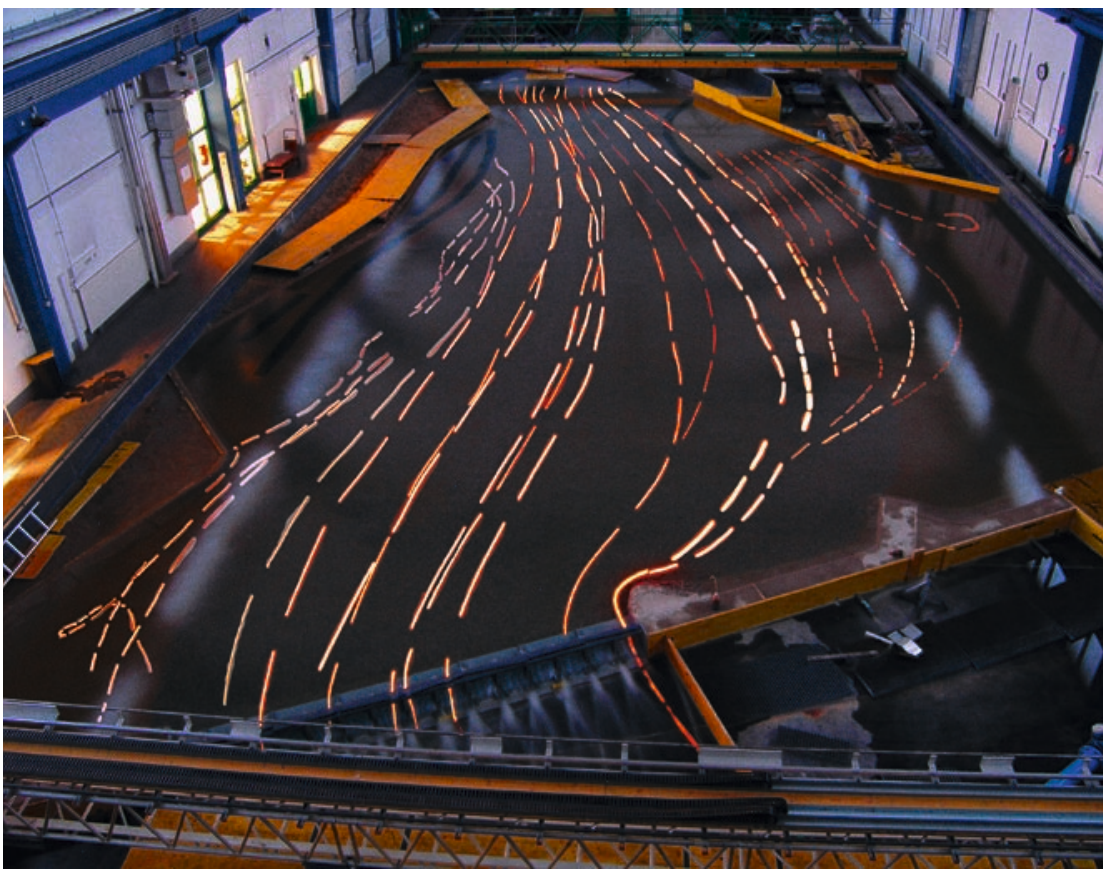

Abb. 13: UW-Ansicht, mit schwimmenden Leuchtkörpern visualisierte Stromlinien und Fließgeschwindigkeiten.

\subsection{Wasserspiegellagen bei $\mathrm{BHQ}=$ $5900 \mathrm{~m}^{3} / \mathrm{s}$}

Im Folgenden werden nur auszugsweise die Messergebnisse mit rauem Flussbett präsentiert, da die Messungen des Wasserspiegels am Vollmodell mit geringer Sohlrauhigkeit überraschenderweise nahezu dieselben Höhen erreichten. Die Ursache lag offensichtlich darin, dass beim Abfluss über das feine Sohlmaterial relativ große Bettumlagerungen mit starker Riffel- und Transportkörperbildung stattfand und damit eine hohe Formrauigkeit wirksam wurde. Beim Abfluss über die relativ raue und stabile Sohle wirkte sich hingegen fast nur die Oberflächenrauigkeit auf das Reibungsgefälle und somit die Wasserspiegellage aus.

Die Messung der Wasserspiegellagen im Stauraum von Ashta 1 beim Bemessungsabfluss (BHQ $=5900 \mathrm{~m}^{3} / \mathrm{s} \div 334 \mathrm{l} / \mathrm{s}$ ) ist zweidimensional in Abbildung 12 dargestellt.

Eine übersichtlichere Gegenüberstellung der Versuchsergebnisse mit den Ergebnissen der numerischen Berechnung findet sich anhand der Wasserspiegellagen in ausgewählten Quer- und Längsprofilen im Punkt 5. Die Auswertungen dieser Messungen und auch der 2d-Simulationen zeigen, dass der Hochwasserspiegel im Stauraum auf ungefähr 27,0 m ü. A. zu liegen kommt. 

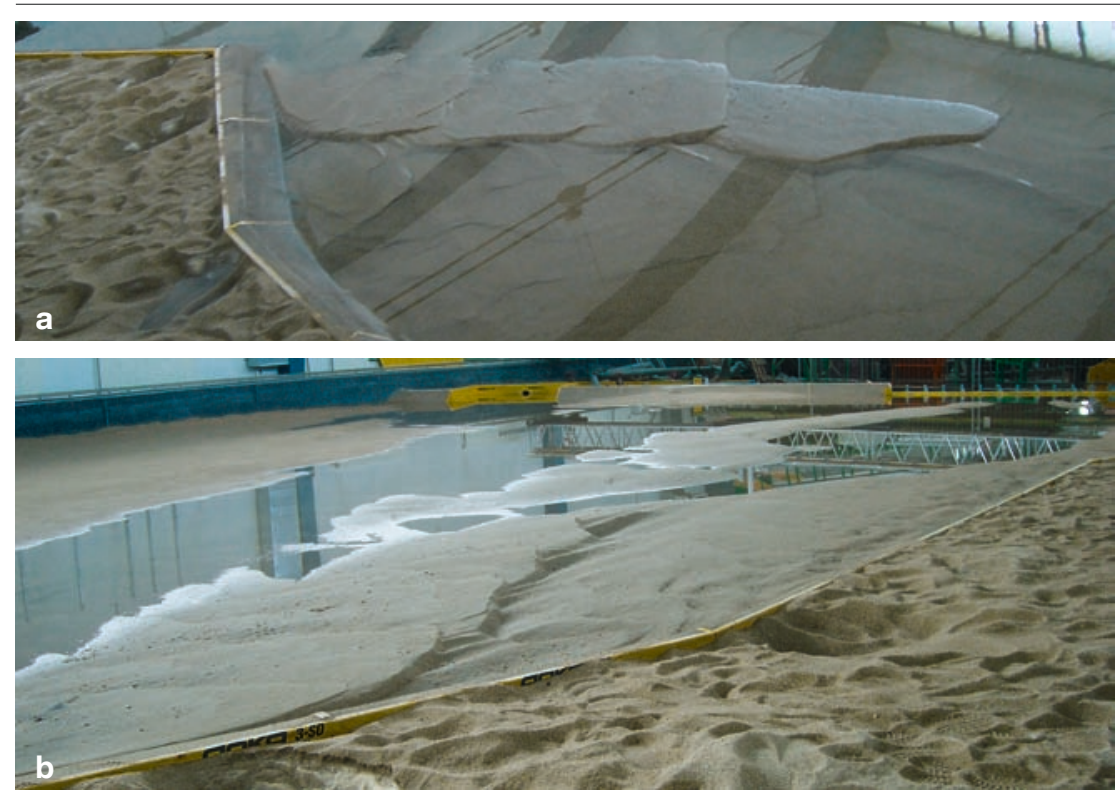

Abb. 14 a, b: Starke Anlandungen an der rechten Seite nach der Zulaufkrümmung, Blick nach OW und UW.

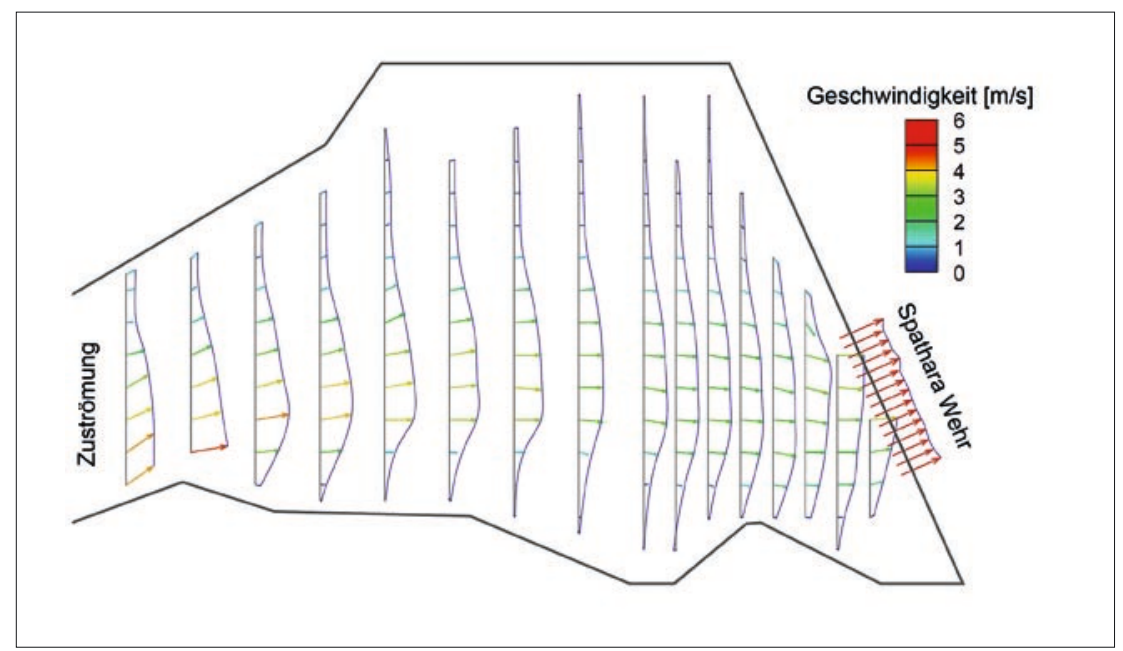

Abb. 15: Fließgeschwindigkeiten (m/s) im physikalischen Vollmodell bei BHQ.

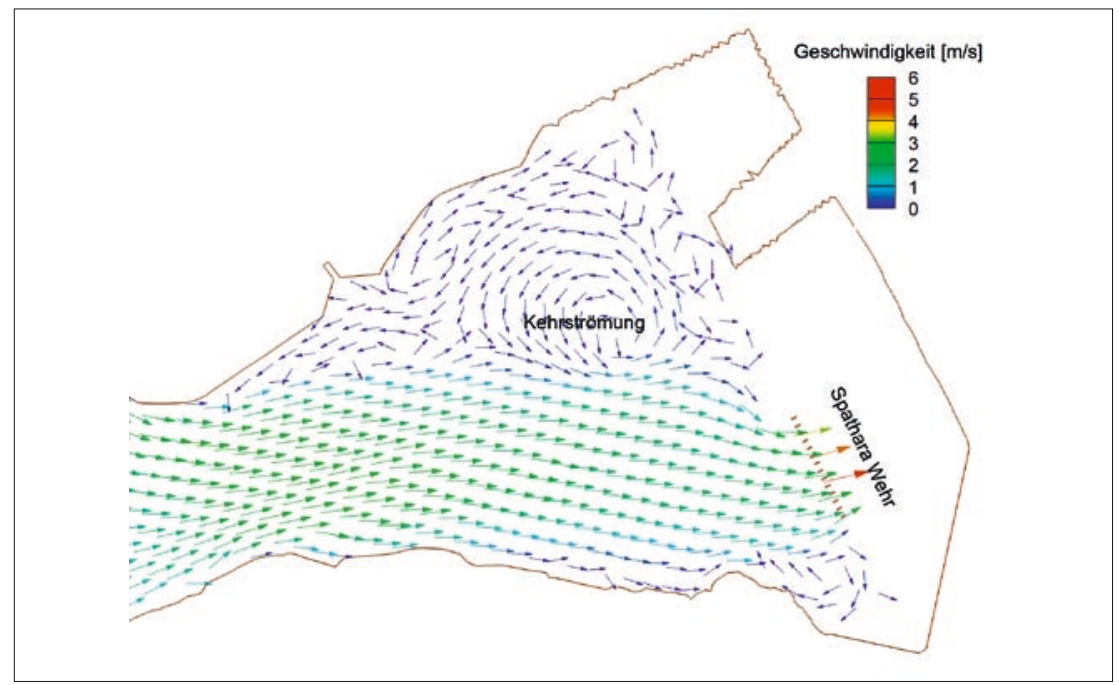

Abb. 16: Berechnete Geschwindigkeitsvektoren beim $B H Q=5900$ m3/s.
4.5. Fließgeschwindigkeiten bei $\mathrm{BHQ}=$ $5900 \mathrm{~m}^{3} / \mathrm{s}$

Das wesentliche Strömungsverhalten während des BHQ-Abflusses im gesamten Modellbereich ist in Abbildung 13 wiedergegeben. Der Hochwasserabfluss erfolgt hauptsächlich in einem Hauptströmungsschlauch direkt in Richtung Spathara Wehr mit einer durchschnittlichen Geschwindigkeit von ca. 3,5 m/s. Die maximale Fließgeschwindigkeit im Stauraum liegt kurz nach dem Einlauf auf der Außenkrümmung in Ufernähe und beträgt ca. $5,5 \mathrm{~m} / \mathrm{s}$ in der Natur. In den Randbereichen des Stauraums findet dagegen kaum Hochwasserabfuhr statt, es kommt sogar links oberhalb des Krafthauszulaufes zu schwachen Rezirkulationsströmungen.

\subsection{Umlagerungen im Staubecken nach $\mathrm{BHQ}$-Abfluss}

Das Flussbett mit der Feinsandausbildung zeigte wie bereits erwähnt im Bereich der Hauptströmung während des BHQ-Abflusses starke Sohlumlagerungen. Die derzeitig vorhandenen Inseln wurden teilweise erodiert, gleichzeitig kam es auch zu neuen Anlandungen. Die im Vorfeld geplanten Baggerungen zur Verbesserung der Wehrzuströmungsverhältnisse im rechten angelandeten Zulaufbereich des Spathara-Wehrs erwiesen sich langfristig als wirkungslos, da es während des HWAbflusses zu einer kompletten Verlandung dieses ausgebaggerten Umlaufkanals kam (Abb. 14).

Nach dem Durchgang einer Hochwasserwelle wird es jedenfalls zu mehr oder weniger großen Anlandungen im Randbereich der Hauptströmung kommen. Fallweise sind daher auch zukünftig Baggerungen im Bereich des Krafthauszulaufes erforderlich um die hydraulischen Verluste bzw. einen Geschiebeeinzug in das Krafthausvorbecken zu reduzieren.

\section{Gegenüberstellung und Diskussion der Ergebnisse}

Die Fließgeschwindigkeiten für den Bemessungsabfluss wurden in 196 Punkten ungefähr $5 \mathrm{~cm}$ unterhalb der Wasseroberfläche gemessen. Der Hochwasserabfluss erfolgt hauptsächlich in einem konzentrierten Hauptkanal in Richtung Spathara Wehr (Abb. 15). Da im 2d-Modell eine etwas höhere Zuströmung aus der Entlastung der Zadeja-Sperre angesetzt wurde, 


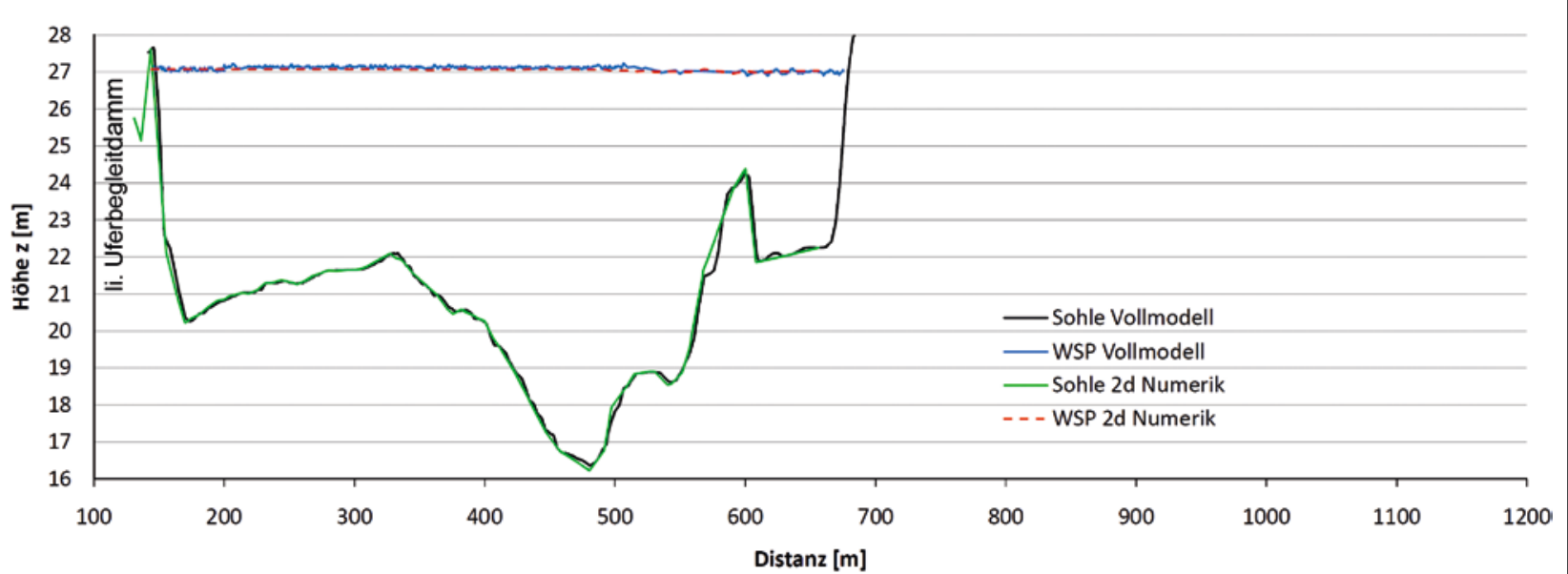

Abb. 17: Wasserspiegellage im Querprofil 1 bei 5900 m³/s, Blick nach UW.

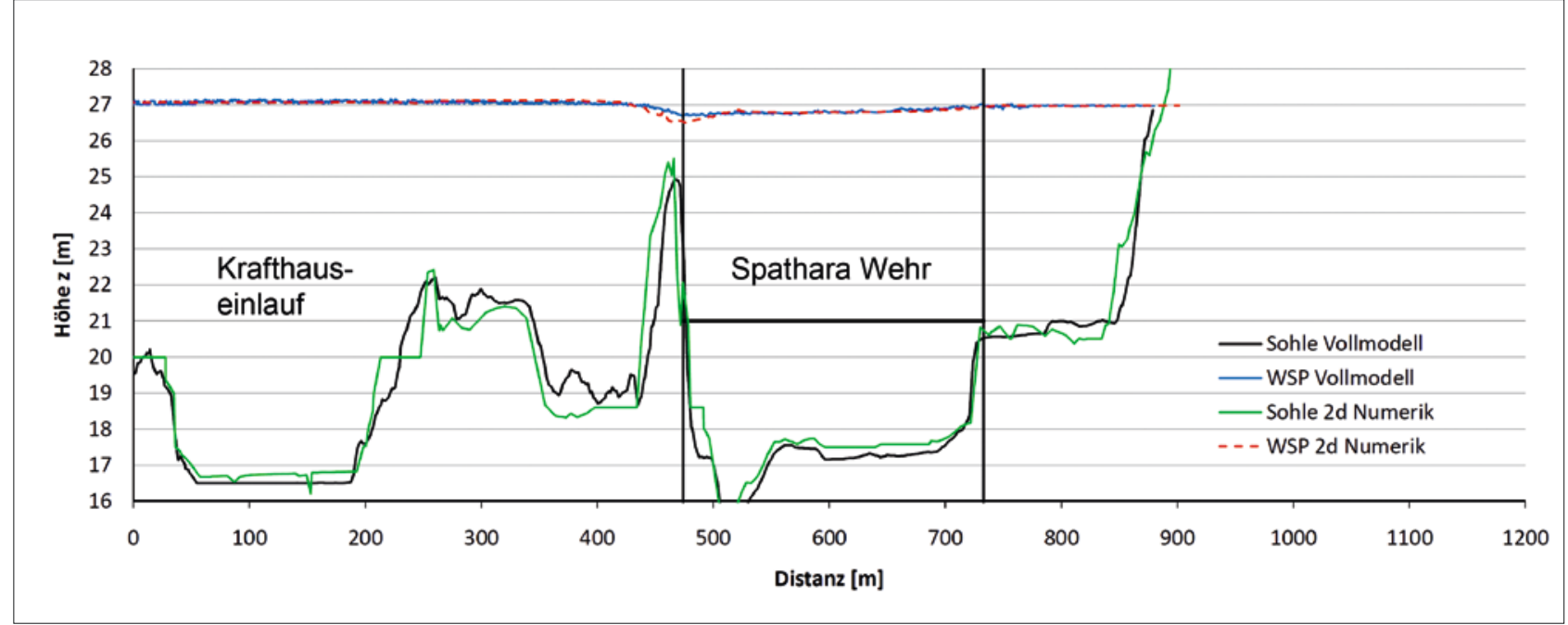

Abb. 18: Wasserspiegellage im Querprofil 2 schräg vor der Wehranlage bei $5900 \mathrm{~m}^{3} / \mathrm{s}$, Blick nach UW.

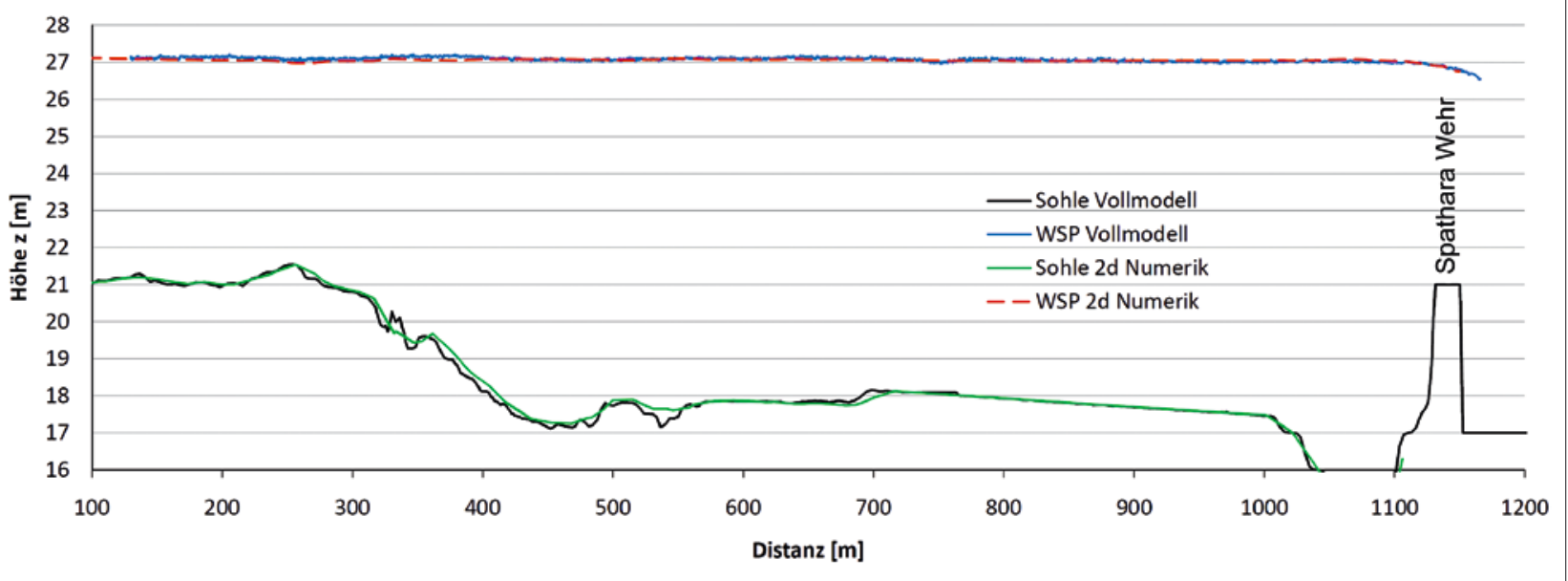

Abb. 19: Wasserspiegellage im Längsprofil bei $5900 \mathrm{~m}^{3} / \mathrm{s}$. 
bildeten sich im Vergleich zum Vollmodell die Geschwindigkeitsmaxima im Außenbogen weniger stark aus. Im Hauptabflussbereich des Stauraums weisen die Geschwindigkeitsvektoren aus der 2d-Berechnung (Abb. 16) im Wesentlichen ein ähnliches Strömungsbild auf wie im Vollmodell.

Die geringfügigen Unterschiede in den Geschwindigkeitsbeträgen können zum Teil im Unterschied von tiefengemittelter Simulation und der oberflächennahen Messung begründet sein. Die Kehrströmungen oberhalb des Kraftwerkszulaufes von Ashtal sind im 2d-Modell stärker ausprägt, als dies in der Leuchtkörperdarstellung des hydraulischen Modells erkennbar ist. Eine Hochwasserabfuhr findet in den Randbereichen kaum statt, es kommt nur zur Ausbildung von Stillwasserzonen mit schwacher Rezirkulation.

Betrachtet man die im Modellversuch gemessenen Wasserspiegellagen und die numerischen Berechnungsergebnisse in den dargestellten Querprofilen ( $A b b .17$, $A b b .18)$ und dem Längsprofil ( $A b b .19$ ), so fällt primär die gute Übereinstimmung der Wasserspiegel auf. Obwohl die Sohlgeometrien nicht vollständig übereinstimmen, zeigen beide Modelluntersuchungen einen etwagleichen Oberwasserspiegel um ca. 27,0 m ü. A. und eine vergleichbare Wasserspiegelabsenkung im Nahbereich des Spathara-Wehrs.

Durch eine entsprechende Wahl der Rauigkeitsverhältnisse (Stricklerbeiwert $\mathrm{k}_{\mathrm{st}}=33$ im Stauraum) in der 2d-Berechnung konnte das Strömungsverhalten (Strömungsbild und Wasserspiegellage) während des Bemessungsabflusses trotz der vorliegenden Einschränkungen sehr gut nachgebildet werden. Ein tatsächlich dreidimensionaler Einfluss der Strömung durch unterschiedliche vertikale und horizontale Geschwindigkeitskomponenten (Anlandungen, variierende Rauigkeiten) hatte offensichtlich geringere Auswirkungen auf die Berechnungsergebnisse als ursprünglich angenommen.

\section{Zusammenfassung und Schlussbemerkung}

Im Zuge der Projektierung der Hydromatrix-Anlage Ashta wurde von Pöyry Energy die numerische Berechnung des Bemessungshochwasserabflusses (BHQ = $5900 \mathrm{~m}^{3} / \mathrm{s}$, entspricht einem geschätzten 500-jährlichen Ereignis) im Stauraum der ersten Stufe Ashta 1 durchgeführt. Im ersten Schritt dienten die Ergebnisse eines hydraulischen Schnittmodellversuchs ( $M=1: 17)$, in dem die Abflussverhältnisse über die mit einem Schlauchwehr aufgerüstete bestehende Wehranlage (SpatharaWehr) bestimmt wurden, als Grundlage für eine zweidimensionale Hochwasserberechnung. In einer weiteren Stufe sollten die Ergebnisse dieser Berechnung anhand eines Vollmodells $(\mathrm{M}=1: 50)$ abgesichert werden, um die Überflutungssicherheit eines bestehenden Begleitdammes sowie der bestehenden Straßen- und Eisenbahnbrücke über die Wehranlage zu gewährleisten. Andernfalls wären weitere Maßnahmen, wie der Bau einer zusätzlichen Hochwasserentlastung, aber auch Baggerungen im Stauraum zur Verbesserung der Anströmverhältnisse der bestehenden Wehranlage, zu setzen.

Mangels zuverlässiger Wasserspiegelaufnahmen während vergangener Hochwasserabflüsse (ein solches ist wenige Monate nach Abschluss der Untersuchungen eingetreten) wurden die Sohlrauigkeiten im Vollmodell stark variiert um ein Spektrum der maximal auftretenden Wasserspiegellagen zu erhalten. Die Messergebnisse wiesen jedoch bei den glatten Modellverhältnissen vermutlich aufgrund starker Sohlumlagerungen ähnliche Höhenlagen auf wie bei jenen mit sehr rauer Sohlausbildung.

Die Strömungsvektoren beim Bemessungshochwasserabfluss zeigten im hydraulischen Vollmodell ein ähnliches Bild, wie es bereits die numerische Berechnung ergab. Dasselbe gilt für die Wasserspiegellagen, die im Bereich des Begleitdammes ca. auf Kote 27,0 mü. A. liegen. Somit werden die geforderten Grenzen, wo zusätzliche bauliche Maßnahmen erforderlich wären, nicht überschritten, obwohl der Untersuchung sehr konservative Annahmen im Bezug auf die Rauigkeitsverhältnisse zugrunde liegen.

Zur Abschätzung des Überflutungsrisikos der Anlage wurde in einer weiterführenden Untersuchung am Vollmodell festgestellt, dass die Überflutung des Begleitdammes und des Brückentragwerkes an der Wehranlage bei einem Abfluss von ca. $7400 \mathrm{~m}^{3} / \mathrm{s}$ über die Wehranlage eintritt, das heißt, der Bemessungsabfluss könnte unter Umständen noch um ca. $25 \%$ überschritten werden, ehe es zum vollständigen Versagen der Anlage kommt. Im Notfall wäre auch eine zusätzliche Hochwasserabfuhr von maximal $720 \mathrm{~m}^{3} / \mathrm{s}$ über den Ausleitungskanal des Kraftwerkes Ashtal möglich.

Abschließend sei angemerkt, dass für eine möglichst naturgetreue Nachbildung von Strömungsproblemen im Wasserbau auch zukünftig eine optimierte Kombination von hydraulischem Experiment und numerischer Berechnung angestrebt werden soll, um zuverlässige Ergebnisse zu erhalten. Je nach Aufgabenstellung führt der gegenwärtige Trend bereits zur Anwendung von hybriden Methoden, wo versucht wird, die Vorteile beider Methoden zu nutzen. Jedenfalls wird der Wasserbauversuch auch in naher Zukunft, trotz sehr großer Fortschritte in der CFD-Simulation, seine Bedeutung als wertvolles Instrument zur Beurteilung von komplexen hydraulischen Aufgabenstellungen und als Ergänzung zur numerischen Strömungsberechnung nicht verlieren.

Korrespondenz:

Ao.Univ.Prof.Dipl.-Ing.Dr.techn.Reinhard Prenner TU Wien

Institut für Wasserbau und Ingenieurhydrologie Karlsplatz 13 A-1040 Wien

Tel.: +43/1/58801-22244

E-Mail: Reinhard.Prenner@kw.tuwien.ac.at 